\title{
PIK3CA somatic alterations in invasive breast cancers: different spectrum from Caucasians to Chinese detected by next generation sequencing
}

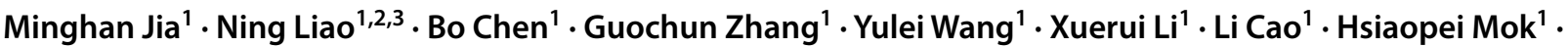 \\ Chongyang Ren ${ }^{1} \cdot$ Kai Li $^{1}$. Cheukfai Li ${ }^{1}$. Lingzhu Wen ${ }^{1}$. Jiali Lin ${ }^{1,3}$. Guangnan Wei ${ }^{1,2}$. Charles M. Balch ${ }^{4}$
}

Received: 15 October 2020 / Accepted: 27 November 2020 / Published online: 1 January 2021

(c) The Author(s) 2021

\begin{abstract}
Purpose Somatic alteration of phosphatidylinositol-4,5-bisphosphate 3-kinase catalytic subunit alpha (PIK3CA) is a crucial therapeutic target in breast cancer (BC) and PI3K $\alpha$-specific inhibitor Alpelisib has been used in clinics. This study investigates the PIK3CA alterations in Chinese and Caucasians BC patients for the purpose of selecting anti-PI3K therapy.

Methods The molecular profile of the PIK3CA gene was analyzed in 412 Chinese patients with untreated invasive BC using a 540 gene next-generation sequencing panel. The results were compared with data of the Caucasian $\mathrm{BC}$ patients in The Cancer Genome Atlas (TCGA-white).

Results PIK3CA alterations were frequently found in BC of estrogen receptor (ER) positive (49.3\%, $p=0.024)$, low ki67 proliferation index $(58.3 \%, p=0.007)$ and low pathological grade (grade I/II/III 80\%, 53.4\%, 35.9\%, $p<0.001)$. Compared to TCGA-white, Chinese BC patients had a higher alteration frequency ( $45.6 \%$ vs. $34.7 \%, p<0.001)$ with larger proportion of p.H1047R mutation among three common mutation sites (p.E545K, p.E542K and p.H1047R) (66.1\% vs. 43.7\%, $p=0.01$ ). Across four molecular subtypes, ER +/human epidermal growth factor receptor 2 positive (HER2 +) tumors harbored the most PIK3CA alterations (51.6\%), while ER-/HER2- harbored the least alteration (30.0\%) but the most copy number amplification $(19.05 \%)$.

Conclusion PIK3CA alterations prevail in Chinese BC patients and have different molecular features compared to that of Caucasians. The results provide precise annotations of PIK3CA genomic alterations of Chinese in the context of application of PIK3CA inhibitor.
\end{abstract}

Keywords PIK3CA $\cdot$ Somatic alteration $\cdot$ Sequencing $\cdot$ Breast cancer $\cdot$ TCGA

\section{Introduction}

Breast cancer (BC) is currently the most common cancer among women worldwide [1]. In addition to the harmful impact by industrialization induced pollutants or mentalrelated endocrine change, $\mathrm{BC}$ is well known as a complex and heterogeneous disease driven by genomic variants [2]. Statistically, $5-10 \%$ of breast cancers are primarily caused

Minghan Jia, Ning Liao, Bo Chen, and Guochun Zhang

contributed equally to this work.

Ning Liao

syliaoning@scut.edu.cn

Extended author information available on the last page of the article by genetic factors resulting from the accumulation of acquired somatic alterations [3].

Somatic alteration of the phosphatidylinositol-4,5-bisphosphate 3-kinase catalytic subunit alpha (PIK3CA) gene is one of the most prevalent driver genes in breast cancer, occurring at a frequency of $20-40 \%[4,5]$. PIK3CA gene is located in chromosome 3q26.32 and encodes the PIK3CA, also named the $\mathrm{p} 110 \alpha$ protein, which is an important component of class I phosphoinositide 3-kinase (PI3K). More than $80 \%$ of PIK3CA mutations occur at three "hotspots", E542K and E545K in exon 9 encoding the helical domains, and H1047R in exon 20 encoding the kinase domains [6]. Through the PI3K/AKT pathway, altered PIK3CA gene plays a critical role in cell survival, apoptosis, proliferation, motility, and adhesion [7]. 
PI3K inhibition as a therapeutic strategy for BC has been investigated throughout many clinical trials [8, 9]. The $\alpha$-specific PI3K inhibitor Alpelisib (PIQRAY, Novartis) has received accelerated approval by the Food and Drug Administration (FDA) in May 2019 for the treatment of patients with estrogen receptor-positive $(\mathrm{HR}+)$ /human epidermal growth factor receptor 2-negative (HER2-) PIK3CA-mutated advanced or metastatic BC based on the SOLAR-1 trial [10]. With the development of next-generation sequencing (NGS) technologies, more recognition on the individual genomic landscape has become possible. Some studies have focused on the altered PIK3CA gene spectrum in different race $[11,12]$. However, there is a lack of comprehensive comparison of the PIK3CA gene alteration spectrum between Caucasians and Chinese in a large scale.

In this study, we report the somatic PIK3CA alterations in 412 Chinese $\mathrm{BC}$ patients, demonstrate the association between the frequency of PIK3CA alterations and the clinicopathological characteristics. We analyze the alteration spectrum within different molecular subgroups, and compare with the data of The Cancer Genome Atlas (TCGA). The ultimate aim is to provide more precise annotations of tumor genomic alterations of Chinese compared to Caucasian in the context of promoting research and application of PI3K inhibitors.

\section{Materials and methods}

\section{Patients and tumor samples}

This study was approved by the ethics committee of Guangdong Provincial People's Hospital (GDPH) [approval number of $2017312 \mathrm{H}(\mathrm{RE})$ ]. The written informed consents were obtained from all participants. A total of 412 Chinese patients of Han nationality who were diagnosed with BC at the GDPH from June 1, 2017 to September 27, 2018 were enrolled in the study. The inclusion criteria are as follows: (1) patients were diagnosed with invasive breast cancer and no chemo or radiotherapy before NGS test; (2) complete clinicopathological information were acquired [sex, age, menstrual status, primary tumor size, axillary lymph node status, pathological type, pathological grade, estrogen receptor (ER), progesterone receptor (PR), and human epidermal growth factor receptor 2 (HER2) status, Ki67 status, distant metastasis status]. Specimens were reviewed by the Department of Pathology at GDPH. The breast cancer molecular subtypes were characterized based on the guideline of St Gallen International Expert Consensus (2019) [13]; and (3) qualified tumor tissue sequencing information.
For The Cancer Genome Atlas (TCGA) cohort, the PIK3CA mutation profiles of Caucasian patients with breast cancer were downloaded from the public website (http:// genome-cancer.ucsc.edu) and analyzed. A total of 453 patients were eligible for this study.

\section{Tissue DNA extraction}

DNA from paraffin-embedded breast cancer tissues was extracted using QIAamp DNA FFPE tissue kit (Qiagen, California, US) according to the manufacturer's instructions. DNA concentration was measured by Qubit dsDNA assay (Life Technologies, California, US).

\section{NGS library preparation and capture-based targeted DNA sequencing}

DNA was subjected to end repair, phosphorylation and adaptor ligation. Fragments of size 200-400 bp were selected by AMPure beads (Agencourt AMPure XP Kit), followed by hybridization with capture probe baits, hybrid selection with magnetic beads and PCR amplification. A high-sensitivity DNA assay was performed to assess the quality and size of the fragments. Indexed samples were sequenced on Nextseq500 sequencer (Illumina, Inc., USA) with pair-end reads.

\section{Sequence data analysis and identification of variants}

Sequence data were mapped to the human genome using BWA aligner 0.7.10. Local alignment optimization, variant calling and annotation were performed using GATK 3.2, MuTect, and VarScan. Variants were filtered using the VarScan fpfilter pipeline, with loci of depth less than 100 filtered out. At least 5 supporting reads were needed for INDELs; while 8 supporting reads were needed for SNVs to be called. According to the ExAC, 1000 Genomes, dbSNP, ESP6500SI-V2 database, variants with population frequency over $0.1 \%$ were grouped as SNP and excluded from further analysis.

The reported mutations were further discerned by searching dbSNP (http://www.ncbi.nlm.nih.gov/ SNP), ClinVar (http://www.ncbi.nlm.nih.gov/clinvar/), COSMIC(http://cancer.sanger.ac.uk/cosmic), BRCA Exchange (http://www.brcaexchange.org/), and Exome Aggregation Consortium (http://exac.broadinstitute.org/) databases along with PubMed publications to detect novel mutations. 


\section{Statistical analysis}

All the computations were performed using the $\mathrm{R}$ software (version3.6.0). Pearson's Chi-square test and Fisher's exact test were used to study the correlation between the clinicopathological features and the occurrence of PIK3CA mutation. The results with obtained $P$ value $<0.05$ were considered statistically significant.

\section{Results}

\section{Association of PIK3CA alterations with clinicopathological features}

We examined a total of 412 tumor tissues of Chinese patients with invasive BC. They were divided into PIK3CA altered $(N=188)$ and wild-type $(N=224)$ groups. The clinicopathological features and PIK3CA gene status of these patients are summarized in Table 1. PIK3CA alterations were predominantly found in $\mathrm{ER}+(49.3 \%, p=0.024)$, low ki67 status $(58.3 \%, p=0.007)$, and low pathological grade tumors (grade I $80 \%$, grade II $53.4 \%$ and grade III $35.9 \%$; $p<0.001)$. The PIK3CA alteration status was significantly different among luminal A (57.6\%), luminal B (44.6\%), Her2-enriched (44.9\%) and basal-like molecular subtypes $(32.1 \%)$. No significant association was found between PIK3CA alterations and onset of age, menopausal status, tumor size, lymph node status, metastasis status, pathological stage, pathological type, PR and Her2 status.

\section{The characteristics of PIK3CA alterations in GDPH and TCGA-white cohort}

In the GDPH cohort, 49 somatic alterations were detected in 188 out of 412 patients, with a gross alteration rate of $45.6 \%$ (Fig. 1a). 163 patients had single alterations (mutation or amplification) and 25 had compound alterations. In the TCGA-white cohort, 43 somatic alterations were detected in 157 out of 453 patients (alteration rate of $34.7 \%$ ) (Fig. 1b), which is lower than that of the GDPH cohort $(p<0.001)$. 127 patients had single alterations and 30 harbored compound mutations.

The PIK3CA alteration type and mutation site were studied and illustrated in Fig. 2. The predominant alteration type was missense mutation (GDPH 90.23\%, TCGA-white 94\%). Both cohorts had similar PIK3CA mutation site. The PIK3CA mutations occurred in several exons (exon1, 2, 4, $5,7-9,13,15,17,19,20)$, with the most frequent location in exon 9 and 20 for both GDPH (73.7\%) and TCGA-white population $(66.0 \%)$. The PIK3CA mutation sites with mutation rate $\geq 1 \%$ in GDPH cohort were showed in Table 2 . The fraction of three common mutation sites including p.E545K,
p.E542K and p.H1047R were showed in Fig. 2c. The TCGAwhite cohort had almost similar common mutations rate among all altered PIK3CA compared to the GDPH cohort (65.6\% vs $66.0 \%, p=0.95)$. However, p.H1047R mutation occupied a significantly higher proportion in GDPH cohort than that of TCGA-white cohort, in all cases $(19.9 \%$ vs $9.9 \%, p<0.001)$ or in common mutations $(66.1 \%$ vs $43.7 \%$, $p=0.01$ ). In addition, 9 novel mutation sites of PIK3CA were detected in the GDPH cohort (Table 3).

\section{Spectrum of PIK3CA alterations across ER/HER2 subtypes}

The PIK3CA alteration spectrum was studied in detail by dividing the GDPH specimens into four ER/HER2 subtypes (Fig. 3). PIK3CA alterations occurred at the highest frequency in ER +/HER $2+(51.6 \%)$ tumors, followed by the ER + /HER2- $(48.7 \%)$ and ER-/HER2 + (43.1\%) tumors, and the lowest in ER-/HER2-(30.0\%) tumors (Table 1). Missense mutation was the primary alteration type for four subtypes, while copy number amplification occupied considerable proportion in ER-/HER2- type (19.05\%). Common mutations distributed extremely alike across subtypes with rates among all altered PIK3CA as $66.1 \%$ in ER + /HER2 -, $66.7 \%$ in ER + /HER +, $64.0 \%$ in ER-/HER2 + and $66.7 \%$ in ER -/HER2 - subtype. Because of the limited sample size, none of the three common mutations were separately analyzed.

\section{Discussion}

In the current study, we explored the frequency and spectra of somatic altered PIK3CA gene by high-resolution genomic sequencing in a large cohort of 412 Chinese patients with invasive $\mathrm{BC}$. To the best of our knowledge, this is one of the largest studies to describe the alteration spectrum of PIK3CA gene in Chinese BC patients of Han nationality compared with Caucasian. The frequency of PIK3CA alteration is $45.6 \%$ in our study, which is slightly higher than that of TCGA-white data (34.7\%) and other literature data (7-36\%) in Chinese [14-16], 14-45\% in other Asians [11, 17, 18], 18-40\% in Americans [19] and 13-45\% in Europeans $[20,21]$. The disparity of PIK3CA gene mutation rate in different studies could be explained by ethnicity, sample size, inclusion criteria or gene sequencing methods.

In accordance with previous studies, the majority of PIK3CA mutations occurred in three hotspot sites, namely E542K and E545K in helical domain and H1047R in kinase domain [11]. Interestingly, there is a significantly larger proportion of p.H1047R mutation in our study than the TCGA Caucasian cohort. The H1047R mutation enhances the interaction of the p110 kinase domain 
Table 1 Clinicopathologic characteristics and PIK3CA status of 412 Chinese patients with invasive breast cancer patients (GDPH cohort)

\begin{tabular}{|c|c|c|c|c|}
\hline Characteristics & & $\begin{array}{l}\text { Wt PIK3CA No.(\%) } \\
(N=224)\end{array}$ & $\begin{array}{l}\text { Altered PIK3CA No.(\%) } \\
(N=188)\end{array}$ & $P$ value \\
\hline \multirow[t]{2}{*}{ Age (years) } & $<=50$ & $132(56.2)$ & $103(43.8)$ & 0.456 \\
\hline & $>50$ & $92(52)$ & $85(48)$ & \\
\hline \multirow[t]{2}{*}{ Sex } & Male & $1(100)$ & 0 & - \\
\hline & Female & $223(54.3)$ & $188(45.7)$ & \\
\hline \multirow[t]{3}{*}{ Menopausal status } & Pre & $129(56.1)$ & $101(43.9)$ & 0.46 \\
\hline & Post & $94(51.9)$ & $87(48.1)$ & \\
\hline & Missing & 1 & 0 & \\
\hline \multirow[t]{4}{*}{ Pathologic T } & $\mathrm{T} 1$ & $80(52.3)$ & 73 (47.7) & 0.303 \\
\hline & $\mathrm{T} 2$ & $115(53.5)$ & $100(46.5)$ & \\
\hline & $\mathrm{T} 3$ & $20(71.4)$ & $8(28.6)$ & \\
\hline & $\mathrm{T} 4$ & $9(56.2)$ & $7(43.8)$ & \\
\hline \multirow[t]{4}{*}{ Pathologic N } & No & $105(52.2)$ & $96(47.8)$ & 0.412 \\
\hline & $\mathrm{N} 1$ & $56(51.9)$ & $52(48.1)$ & \\
\hline & $\mathrm{N} 2$ & $44(59.5)$ & $30(40.5)$ & \\
\hline & N3 & $19(65.5)$ & $10(34.5)$ & \\
\hline \multirow[t]{3}{*}{ Pathologic M } & M0 & 207 (53.9) & $177(46.1)$ & 0.476 \\
\hline & M1 & $17(63)$ & $10(37)$ & \\
\hline & Missing & 0 & 1 & \\
\hline \multirow[t]{8}{*}{ Pathologic stage } & IA & $49(48)$ & $53(52)$ & 0.468 \\
\hline & IIA & $71(59.2)$ & $49(40.8)$ & \\
\hline & IIB & $36(50.7)$ & $35(49.3)$ & \\
\hline & IIIA & $31(53.4)$ & 27 (46.6) & \\
\hline & IIIB & $3(42.9)$ & $4(57.1)$ & \\
\hline & IIIC & $17(65.4)$ & $9(34.6)$ & \\
\hline & IV & $17(63)$ & $10(37)$ & \\
\hline & Missing & 0 & 1 & \\
\hline \multirow[t]{4}{*}{ Pathological grade } & I & $3(20)$ & $12(80)$ & $<0.001$ \\
\hline & II & 89 (46.6) & $102(53.4)$ & \\
\hline & III & $127(64.1)$ & $71(35.9)$ & \\
\hline & Missing & 5 & 3 & \\
\hline \multirow[t]{3}{*}{ Pathological type } & Ductal & $193(53.5)$ & $168(46.5)$ & 0.184 \\
\hline & Lobular & $4(30.8)$ & $9(69.2)$ & \\
\hline & Others & $27(71.1)$ & $11(28.9)$ & \\
\hline \multirow[t]{2}{*}{ ER status } & Positive & $149(50.7)$ & $145(49.3)$ & 0.024 \\
\hline & Negative & 75 (63.6) & $43(36.4)$ & \\
\hline \multirow[t]{2}{*}{ PR status } & Positive & $143(52.4)$ & $130(47.6)$ & 0.303 \\
\hline & Negative & $81(58.3)$ & $58(41.7)$ & \\
\hline \multirow[t]{3}{*}{ HER2 status } & Positive & $60(51.3)$ & $57(48.7)$ & 0.306 \\
\hline & Negative & 157 (57.5) & $116(42.5)$ & \\
\hline & Missing & 7 & 15 & \\
\hline \multirow[t]{3}{*}{ Ki67 status } & $<14$ & $40(41.7)$ & $56(58.3)$ & 0.007 \\
\hline & $>=14$ & $182(58)$ & $132(42)$ & \\
\hline & Missing & 2 & 0 & \\
\hline \multirow[t]{5}{*}{ Molecular subtype } & Luminal A & $36(42.4)$ & $49(57.6)$ & 0.03 \\
\hline & Luminal B & $124(55.4)$ & $100(44.6)$ & \\
\hline & HER2-enriched & $27(55.1)$ & $22(44.9)$ & \\
\hline & Basal-like & $36(67.9)$ & $17(32.1)$ & \\
\hline & Missing & 1 & 0 & \\
\hline \multirow[t]{4}{*}{ ER/HER2 subtype } & ER +/HER2- & 118(51.3) & 112(48.7) & 0.05 \\
\hline & $\mathrm{ER}+/ \mathrm{HER} 2+$ & $31(48.4)$ & $33(51.6)$ & \\
\hline & ER-/HER2+ & $33(56.9)$ & $25(43.1)$ & \\
\hline & ER-/HER2- & $42(70.0)$ & $18(30.0)$ & \\
\hline
\end{tabular}


Table 1 (continued)
GDPH Guangdong Provincial People's Hospital, No. number, Wt wild type, PIK3CA phosphatidylinositol4,5-bisphosphate 3-kinase catalytic subunit alpha, $E R$ estrogen receptor, $P R$ progesterone receptor, $H E R 2$ human epidermal growth factor receptor 2
A

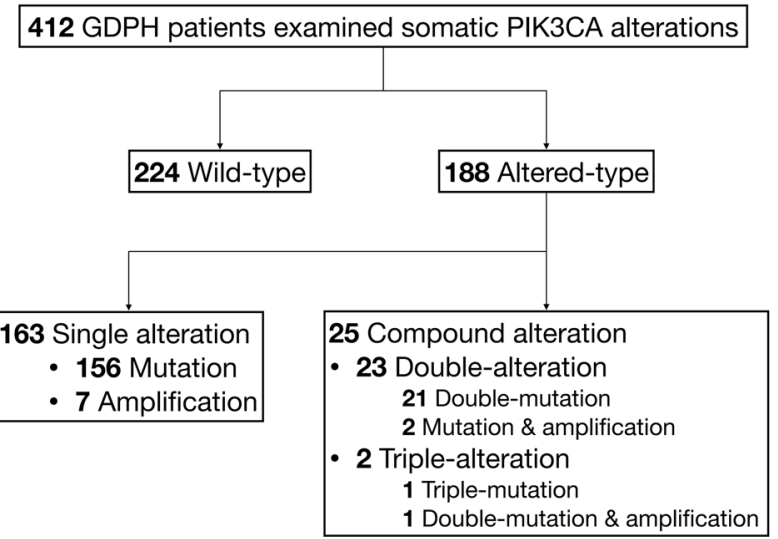

Fig. 1 PIK3CA somatic alteration distribution across breast cancer population of GDPH and TCGA-white cohorts. a Distribution in GDPH population. b Distribution in TCGA-white popu-
B

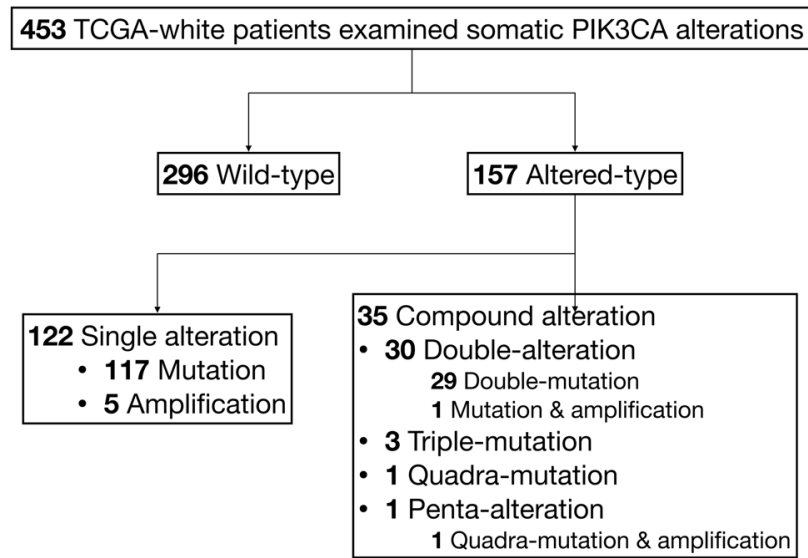

lation. PIK3CA phosphatidylinositol-4,5-bisphosphate 3-kinase catalytic subunit alpha, GDPH Guangdong Provincial People's Hospital, TCGA The Cancer Genome Atlas
A

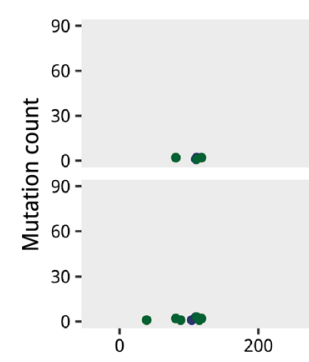

D

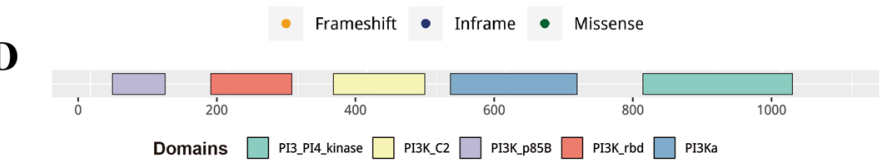

B

C

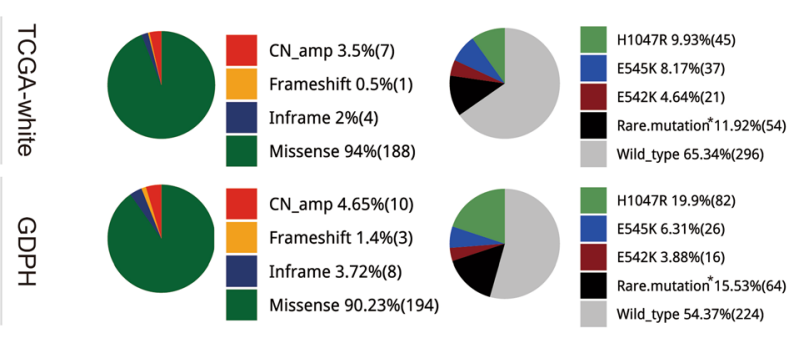

Fig. 2 PIK3CA alteration spectrum in breast cancer of the TCGA and GDPH cohorts. a Distribution of mutation across the gene. b Pie charts showing the fraction of alteration types. $\mathbf{c}$ Pie charts showing the fraction of mutation sites. $\mathbf{d}$ Conserved and functional domains of PIK3CA gene. *Rare mutation: defined as PIK3CA mutations except

with cell membranes, while the E542K and E545K mutations disrupt the inhibitory interface with p85 [22]. Due to different functional mechanisms, 3 hotspot sites may cause different damage through downstream signaling. An in vitro study showed that the helical domain mutants produced a more aggressive phenotype than kinase domain mutants [23]. However, one study suggested that the mere existence of mutant E545K may not be as harmful as H1047R [24]. As for utilization of antineoplastic drugs, three common mutation sites: p.E545K, p.E542K and p.H1047R. PIK3CA phosphatidylinositol-4,5-bisphosphate 3-kinase catalytic subunit alpha, GDPH Guangdong Provincial People's Hospital, TCGA The Cancer Genome Atlas, CN_amp copy number amplification

limited studies have focused on the relationship between specific PIK3CA alteration site and effectiveness of drugs [25]. In a retrospective analysis, Binghe $\mathrm{Xu}$, etc. suggested that PIK3CA/H1047R mutations may be a potential biomarker of sensitivity to everolimus, wherein H1047R mutated patients had longer PFS than wild-type or other mutant forms of PIK3CA. Analyses in the neoadjuvant setting suggested that mutations in exon 9 conferred a higher sensitivity to pictilisib when compared with mutations in 
Table 2 PIK3CA mutation sites with mutation rate $\geq 1 \%$ in GDPH cohort and corresponding rate in TCGAwhite cohort

\begin{tabular}{lllllr}
\hline Exon & Nucleotide change & Mutation site & $\begin{array}{l}\text { GDPH No. }(\%) \\
(N=412)\end{array}$ & $\begin{array}{l}\text { TCGA-white } \\
\text { No. }(\%) \\
(N=453)\end{array}$ & $P$ value \\
\hline 20 & c.3140 A > G & p.H1047R & $82(19.9)$ & $45(9.9)$ & $<\mathbf{0 . 0 0 1}$ \\
9 & c.1633 G > A & p.E545K & $27(6.6)$ & $37(8.2)$ & 0.435 \\
9 & c.1624 G > A & p.E542K & $17(4.1)$ & $21(4.6)$ & 0.743 \\
4 & c.1035 T > A & p.N345K & $14(3.4)$ & $8(1.8)$ & 0.137 \\
20 & c.3140 A > T & p.H1047L & $8(1.9)$ & $8(1.8)$ & 1.000 \\
13 & c. $2176 \mathrm{G}>$ A & p.E726K & $6(1.5)$ & $4(0.9)$ & - \\
7 & c.1258 T > C & p.C420R & $4(1)$ & $1(0.2)$ & - \\
\hline
\end{tabular}

PIK3CA phosphatidylinositol-4,5-bisphosphate 3-kinase catalytic subunit alpha, GDPH Guangdong Provincial People's Hospital, TCGA The Cancer Genome Atlas, $p$. protein sequence, No. number
Table 3 Novel mutation sites of PIK3CA detected in GDPH cohort

\begin{tabular}{llll}
\hline Mutation site & Codon & Mutation type & Exon \\
\hline p.V344_V346dup & 344 & Inframe & 4 \\
p.E418_L422 > V & 418 & Inframe & 7 \\
p.P449_E453 > Q & 449 & Inframe & 7 \\
p.P449_V461del & 449 & Inframe & 7 \\
p.P449S & 449 & Missense & 7 \\
p.E798N & 798 & Missense & 15 \\
p.Q861fs & 861 & Frameshift & 17 \\
p.E976K & 976 & Missense & 19 \\
p.A1066fs & 1066 & Frameshift & 20 \\
\hline
\end{tabular}

PIK3CA phosphatidylinositol-4,5-bisphosphate 3-kinase catalytic subunit alpha, GDPH Guangdong Provincial People's Hospital, TCGA The Cancer Genome Atlas, $p$. protein sequence, No. number

exon 20 [26]. In general, two puzzles remain to be clarified about clinical significance of PIK3CA alteration sites: (1) extent of harmfulness for different PIK3CA alteration domains; and (2) PIK3CA alteration site as a biomarker for patients who benefit most from PI3K inhibitors.

Another question is the relationship between PIK3CA alterations and clinicopathological characteristics. In present study, PIK3CA alterations happen more in BC with low pathological grade, ER positive and low Ki67 index. The positive correlation between PIK3CA alteration and better prognosis has also been previously reported [20, 27, 28]. A large-scale systematic review of BC clinical studies involved 2587 cases showed that patients with tumors harboring a PIK3CA mutation have a better clinical outcome, especially for postmenopausal women with ER + BC [29]. However, recent molecular profiling data from $\mathrm{MBC}$ patients seem to indicate that in advanced HR +/HER2 - BC, a PIK3CA mutation would lead to resistance to chemotherapy and a poor outcome [30]. On all accounts, improved understanding of the clinical impact of PIK3CA alterations is critical to prevent or explain therapeutic failures and develop optimal personalized therapeutics against breast cancer.

Additionally, the relevance between PIK3CA alteration spectrum and ER/HER2 molecular subtypes has also been detected in our study. PIK3CA alterations were more prevalent in ER + tumors and the least in the ER-/HER2-(30.0\%) subtype, in agreement with previous studies [5, 21, 31]. Despite the overall low prevalence of PIK3CA mutations in TNBC, the proportion is not negligible. The finding of Jiang etc. [4] stated that Chinese TNBC cases demonstrate $18 \%$ PIK3CA mutations, we found the mutation rate of $30 \%$. Therefore, high PIK3CA mutation may be a remarkable feature of Chinese TNBC patients. Meanwhile, ER-/HER2- subtype in our study is enriched for frameshift and DNA copy number amplification, which was similarly observed previously [32], suggesting the genomic instability in TNBC subtypes. Several clinical studies are trying to determine the potential benefit of PIK3CA inhibitors in different BC subtypes. Although the efficacy of additional PIK3CA inhibitor shows lack of significance in the early setting [33,34], the combined use with existing therapies in heavily pretreated MBC patients demonstrates substantial prospective [10, 35]. On all accounts, a biomarker-driven approach to identify optimal clinical choices in the background of PIK3CA gene alterations is warranted.

In conclusion, our study showed that PIK3CA alteration is a common event in Chinese $\mathrm{BC}$ patients and has a unique spectrum compared to that of Caucasians. The distribution patterns of PIK3CA alteration are distinct across four molecular subtypes based on ER/HER status. Future clinical trials should assess PIK3CA alteration characteristics as biomarkers to predict therapeutic response to PI3K inhibitors. 
A

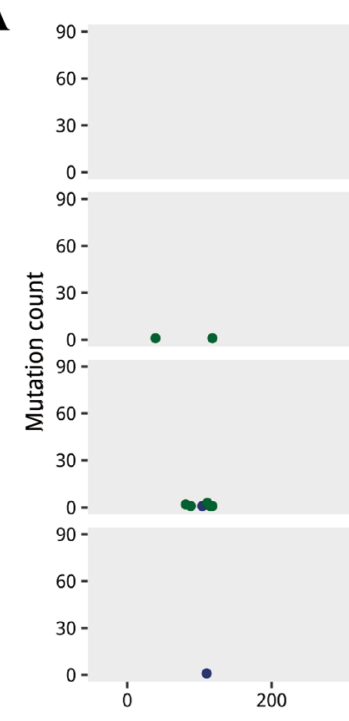

C

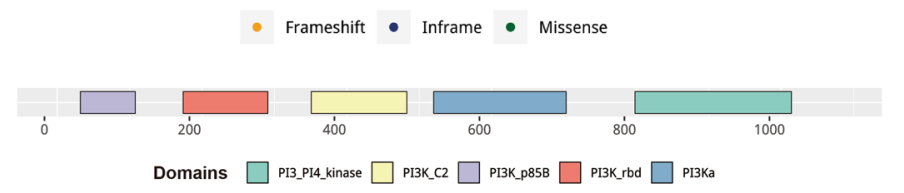

B 涌

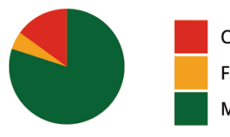

CN_amp 15\%(3)

Frameshift 5\%(1)

Missense $80 \%(16)$

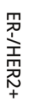

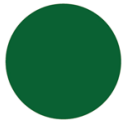

Missense $100 \%(22$

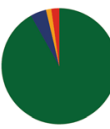

CN_amp 2.22\%(3)

Frameshift $1.48 \%(2)$

Inframe $4.44 \%(6)$

Missense $91.85 \%(124)$

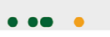

罜

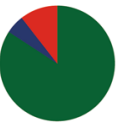

CN_amp $10.53 \%(4)$

Inframe $5.26 \%(2)$

Missense $84.21 \%(32)$
Fig. 3 PIK3CA alteration spectrum across four ER/HER2 subgroups in breast cancer of GDPH cohort. A. Distribution of mutation across the gene. B. Pie charts showing the fraction of alteration types. C. Conserved and functional domains of PIK3CA gene. PIK3CA phos-

Acknowledgements We thank all the patients and their families for participation. This study was supported by funding from National Natural Science Foundation of China (81602645; 81071851; 81001189), Natural Science Foundation of Guangdong Province (2016A030313768; 2018A030313292) and Research Funds from Guangzhou Municipal Science and Technology Project $(201707010418 ; 201804010430)$

Funding The funding body has no roles in the design of the study and collection, analysis and interpretation of data and in writing the manuscript.

\section{Compliance with ethical standards}

Conflict of interest The authors declare no competing financial interests.

Open Access This article is licensed under a Creative Commons Attribution 4.0 International License, which permits use, sharing, adaptation, distribution and reproduction in any medium or format, as long as you give appropriate credit to the original author(s) and the source, provide a link to the Creative Commons licence, and indicate if changes were made. The images or other third party material in this article are included in the article's Creative Commons licence, unless indicated otherwise in a credit line to the material. If material is not included in the article's Creative Commons licence and your intended use is not permitted by statutory regulation or exceeds the permitted use, you will need to obtain permission directly from the copyright holder. To view a copy of this licence, visit http://creativecommons.org/licenses/by/4.0/. phatidylinositol-4,5-bisphosphate 3-kinase catalytic subunit alpha, GDPH Guangdong Provincial People's Hospital, TCGA The Cancer Genome Atlas, $C N \_a m p$ copy number amplification

\section{References}

1. Yap YS, Lu YS, Tamura K, Lee JE, Ko EY, Park YH, et al. Insights into breast cancer in the east vs the west: a review. JAMA Oncol. 2019;5(10):1489-96.

2. Akram M, Iqbal M, Daniyal M, Khan AU. Awareness and current knowledge of breast cancer. Biol Res. 2017;50:33.

3. Couch FJ, Nathanson KL, Offit K. Two decades after BRCA: setting paradigms in personalized cancer care and prevention. Science. 2014;343:1466-70.

4. Jiang YZ, Ma D, Suo C, Shi J, Xue M, Hu X, et al. Genomic and transcriptomic landscape of triple-negative breast cancers: subtypes and treatment strategies. Cancer Cell. 2019;35:428-40 (e5).

5. Martínez-Sáez O, Chic N, Pascual T, Adamo B, Vidal M, González-Farré B, et al. Frequency and spectrum of PIK3CA somatic mutations in breast cancer. Breast Cancer Res. 2020;22:45.

6. Arafeh R, Samuels Y. PIK3CA in cancer: the past 30 years. Semin Cancer Biol. 2019;59:36-49.

7. Goncalves MD, Hopkins BD, Cantley LC. Phosphatidylinositol 3-kinase, growth disorders, and cancer. N Engl J Med. 2018;379:2052-62.

8. Dickler MN, Saura C, Richards DA, Krop IE, Cervantes A, Bedard PL. Phase II study of taselisib (GDC-0032) in combination with fulvestrant in patients with HER2-negative, hormone receptorpositive. Adv Breast Cancer. 2018;24:4380-7.

9. Baselga J, Im SA, Iwata H, Cortes J, De Laurentiis M, Jiang Z, et al. Buparlisib plus fulvestrant versus placebo plus fulvestrant 
in postmenopausal, hormone receptor-positive, HER2-negative, advanced breast cancer (BELLE-2): a randomised, double-blind, placebo-controlled, phase 3 trial. Lancet Oncol. 2017;18:904-16.

10. Andre F, Ciruelos E, Rubovszky G, Campone M, Loibl S, Rugo HS, et al. Alpelisib for PIK3CA-mutated, hormone receptor-positive advanced breast cancer. N Engl J Med. 2019;380:1929-40.

11. Azizi Tabesh G, Izadi P, Fereidooni F, Emami Razavi AN, Tavakkoly BJ. The high frequency of PIK3CA mutations in iranian breast cancer patients. Cancer Invest. 2016;35:36-42.

12. Ahmad F, Badwe A, Verma G, Bhatia S, Das BR. Molecular evaluation of PIK3CA gene mutation in breast cancer: determination of frequency, distribution pattern and its association with clinicopathological findings in Indian patients. Med Oncol. 2016;33:74.

13. Burstein HJ, Curigliano G, Loibl S, Dubsky P, Gnant M, Poortmans $\mathrm{P}$, et al. Estimating the benefits of therapy for early-stage breast cancer: the St. Gallen International Consensus Guidelines for the primary therapy of early breast cancer 2019. Ann Oncol. 2019;30:1541-57.

14. Tong L, Yang XX, Liu MF, Yao GY, Dong JY, Ye CS, et al. Mutational analysis of key EGFR pathway genes in Chinese breast cancer patients. Asian Pac J Cancer Prev. 2012;13:5599-603.

15. Li G, Guo X, Chen M, Tang L, Jiang H, Day JX, et al. Prevalence and spectrum of AKT1, PIK3CA, PTEN and TP53 somatic mutations in Chinese breast cancer patients. PLoS ONE. 2018;13:e0203495.

16. Liu S, Wang H, Zhang L, Tang C, Jones L, Ye H, et al. Rapid detection of genetic mutations in individual breast cancer patients by next-generation DNA sequencing. Hum Genomics. 2015;9:2.

17. Takeshita T, Yamamoto Y, Yamamoto-Ibusuki M, Inao T, Sueta A, Fujiwara S, et al. Prognostic role of PIK3CA mutations of cellfree DNA in early-stage triple negative breast cancer. Cancer Sci. 2015;106:1582-9.

18. Sudhakar N, Priya Doss CG, Thirumal Kumar D, Chakraborty C, Anand K, Suresh M. Deciphering the impact of somatic mutations in exon 20 and exon 9 of PIK3CA gene in breast tumors among Indian women through molecular dynamics approach. J Biomol Struct Dyn. 2016;34:29-41.

19. Pereira B, Chin SF, Rueda OM, Vollan HK, Provenzano E, Bardwell HA, et al. The somatic mutation profiles of 2433 breast cancers refines their genomic and transcriptomic landscapes. Nat Commun. 2016;7:11479.

20. Boyault S, Drouet Y, Navarro C, Bachelot T, Lasset C, Treilleux I, et al. Mutational characterization of individual breast tumors: TP53 and PI3K pathway genes are frequently and distinctively mutated in different subtypes. Breast Cancer Res Treat. 2012;132:29-39.

21. Cizkova M, Susini A, Vacher S, Cizeron-Clairac G, Andrieu C, Driouch K, et al. PIK3CA mutation impact on survival in breast cancer patients and in ERalpha, PR and ERBB2-based subgroups. Breast Cancer Res. 2012;14:R28.

22. Fruman DA, Chiu H, Hopkins BD, Bagrodia S, Cantley LC, Abraham RT. The PI3K pathway in human disease. Cell. 2017;170:605-35.

23. Pang H, Flinn R, Patsialou A, Wyckoff J, Roussos ET, Wu H, et al. Differential enhancement of breast cancer cell motility and metastasis by helical and kinase domain mutations of class IA phosphoinositide 3-kinase. Cancer Res. 2009;69:8868-76.

24. Thirumal Kumar D, George Priya Doss C. Role of E542 and E545 missense mutations of PIK3CA in breast cancer: a comparative computational approach. J Biomol Struct Dyn. 2017;35:2745-57.
25. Brandão M, Caparica R, Eiger D, de Azambuja E. Biomarkers of response and resistance to $\mathrm{PI} 3 \mathrm{~K}$ inhibitors in estrogen receptorpositive breast cancer patients and combination therapies involving PI3K inhibitors. Ann Oncol. 2019;30(Suppl 10):x27-42.

26. Schmid P, Pinder SE, Wheatley D, Macaskill J, Zammit C, Hu J, et al. Phase II randomized preoperative window-of-opportunity study of the PI3K inhibitor pictilisib plus anastrozole compared with anastrozole alone in patients with estrogen receptor-positive breast cancer. J Clin Oncol. 2016;34:1987-94.

27. Arsenic R, Treue D, Lehmann A, Hummel M, Dietel M, Denkert $\mathrm{C}$, et al. Comparison of targeted next-generation sequencing and Sanger sequencing for the detection of PIK3CA mutations in breast cancer. BMC Clin Pathol. 2015;15:20.

28. Wu H, Wang W, Du J, Li H, Wang H, Huang L, et al. The distinct clinicopathological and prognostic implications of PIK3CA mutations in breast cancer patients from Central China. Cancer Manag Res. 2019;11:1473-92.

29. Dumont AG, Dumont SN, Trent JC. The favorable impact of PIK3CA mutations on survival: an analysis of 2587 patients with breast cancer. Chin J Cancer. 2012;31:327-34.

30. Mosele F, Stefanovska B, Lusque A, Tran Dien A, Garberis I, Droin N, et al. Outcome and molecular landscape of patients with PIK3CA-mutated metastatic breast cancer. Ann Oncol. 2020;31:377-86.

31. Howlader N, Altekruse SF, Li CI, Chen VW, Clarke CA, Ries LA, et al. US incidence of breast cancer subtypes defined by joint hormone receptor and HER2 status. J Natl Cancer Inst. 2014;106(5): 1.

32. Natrajan R, Weigelt B, Mackay A, Geyer FC, Grigoriadis A, Tan DS, et al. An integrative genomic and transcriptomic analysis reveals molecular pathways and networks regulated by copy number aberrations in basal-like, HER 2 and luminal cancers. Breast Cancer Res Treat. 2010;121:575-89.

33. Mayer IA, Prat A, Egle D, Blau S, Fidalgo JAP, Gnant M, et al. A phase II randomized study of neoadjuvant letrozole plus alpelisib for hormone receptor-positive, human epidermal growth factor receptor 2-negative breast cancer (NEO-ORB). Clin Cancer Res. 2019;25:2975-87.

34. Saura C, Hlauschek D, Oliveira M, Zardavas D, Jallitsch-Halper $A$, de la Peña L, et al. Neoadjuvant letrozole plus taselisib versus letrozole plus placebo in postmenopausal women with oestrogen receptor-positive, HER2-negative, early-stage breast cancer (LORELEI): a multicentre, randomised, double-blind, placebocontrolled, phase 2 trial. Lancet Oncol. 2019;20:1226-38.

35. Jain S, Shah AN, Santa-Maria CA, Siziopikou K, Rademaker A, Helenowski I, et al. Phase I study of alpelisib (BYL-719) and trastuzumab emtansine (T-DM1) in HER2-positive metastatic breast cancer (MBC) after trastuzumab and taxane therapy. Breast Cancer Res Treat. 2018;171:371-81.

Publisher's Note Springer Nature remains neutral with regard to jurisdictional claims in published maps and institutional affiliations. 


\section{Affiliations}

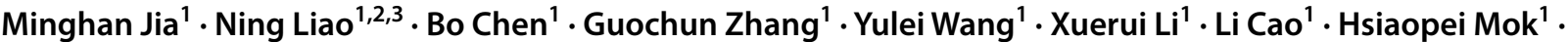

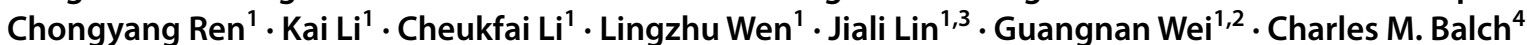

Minghan Jia

328820736@qq.com

Bo Chen

chenb45@mail2.sysu.edu.cn

Guochun Zhang

wincu99@163.com

Yulei Wang

yuleiwang@whu.edu.cn

Xuerui Li

mxlxr@163.com

$\mathrm{LiCaO}$

constancetsau@126.com

Hsiaopei Mok

765740693@qq.com

Chongyang Ren

568306417@qq.com

Kai Li

11111230013@fudan.edu.cn

Cheukfai Li

cheukfaili@163.com
Lingzhu Wen

dearecho@msn.com

Jiali Lin

13414057347@163.com

Guangnan Wei

dianasalice@sina.com

Charles M. Balch

CMBalch@mdanderson.org

1 Department of Breast Cancer, Cancer Center, Guangdong Provincial People's Hospital, Guangdong Academy of Medical Sciences, 106 Zhongshan Er Road, Guangzhou 510080, Guangdong, China

2 School of Medicine, South China University of Technology, Guangzhou, China

3 The Second School of Clinical Medicine, Southern Medical University, Guangzhou, China

4 Department of Surgical Oncology, The University of Texas MD Anderson Cancer Center, Houston, TX, USA 